\title{
On the minimum number of edges giving maximum oriented chromatic number
}

\author{
Alexandr V. Kostochka* \\ Gábor Simonyi ${ }^{\ddagger}$
}

\author{
Tomasz Łuczak ${ }^{\dagger}$ \\ Eric Sopena ${ }^{\S}$
}

\begin{abstract}
We show that the minimum number of edges in a graph on $n$ vertices with oriented chromatic number $n$ is $(1+o(1)) n \log _{2} n$.
\end{abstract}

In 1995, in a conversation with the French member of the set of the authors of this note, Pál Erdős asked about the minimal number of edges a graph on $n$ vertices with oriented chromatic number $n$ can have. During the conference on the Future of Discrete Mathematics in the cosy but fruitful atmosphere of the Štiř́n Castle we found an elementary answer to this question which we present below. ${ }^{1}$

${ }^{*}$ Novosibirsk State University, Novosibirsk, Russia 630090. Research partially supported by the grant 96-01-01614 of the Russian Foundation for Fundamental Research and by the Cooperative Grant Award RM1-181 of the US Civilian Research and Development Foundation.

†Department of Discrete Mathematics, Adam Mickiewicz University, 60-769 Poznań, Poland. Research partially supported by KBN grant 2 P03A 02309.

$\ddagger$ Mathematical Institute of the Hungarian Academy of Sciences, P.O.B.127, Budapest H-1364, Hungary. Research partially supported by the Hungarian National Foundation for Scientific Research (OTKA) Grant Nos. F023442 and T016386.

$\S$ LaBRI, Université Bordeaux I, 33405 Talence Cedex, France. Research partially supported by the Barrande Grant no. 97137.

${ }^{1}$ After the final version of this note had been sent to the publisher we were informed that a very similar result had been proved independently by Z. Füredi, P. Horak, C. M. Pareek and X. Zhu in the paper Minimal oriented graphs of diameter 2, which is to appear in Graphs and Combinatorics. 
Let us consider first an extremal question closely related to Erdős's original problem. A colouring of the vertices of a graph $G$ whose edges are initially coloured with $k$ colours is admissible, if every colour class spans an independent set and each pair of colour classes is joined by edges of one colour only. After Alon and Marshall [1], we define the $k$-chromatic number $\chi_{k}(G)$ of $G$ as the minimal number $\ell$ such that for any colouring of edges of $G$ with $k$ colours there exists an admissible $\ell$-colouring of the vertices of $G$. Finally, by $f_{k}(n)$ we denote the minimum number of edges in a graph $G$ on $n$ vertices for which $\chi_{k}(G)=n$. We shall show that for any fixed $k$ the function $f_{k}(n)$ grows roughly as $n \log _{k} n$.

Theorem 1. For a fixed $k \geq 2$ and $n$ large enough

$$
n\left(\log _{k} n-4 \log _{k} \log _{k} n-5\right) \leq f_{k}(n) \leq\left\lceil\log _{k} n\right\rceil\left(n-\left\lceil\log _{k} n\right\rceil\right) .
$$

Proof of Theorem 1. In order to see the upper bound for $f_{k}(n)$ observe that the complete bipartite graph on $n$ vertices, with bipartition $\left(W^{\prime}, W^{\prime \prime}\right)$, where $\left|W^{\prime}\right|=\left\lceil\log _{k} n\right\rceil$ and $\left|W^{\prime \prime}\right|=n-\left\lceil\log _{k} n\right\rceil$, has $k$-chromatic number $n$. Indeed, label elements of $W^{\prime}$ by $1, \ldots,\left\lceil\log _{k} n\right\rceil$ and elements of $W^{\prime \prime}$ by $0,1, \ldots, n-$ $\left\lceil\log _{k} n\right\rceil-1$. Now colour the edge $\left\{i^{\prime}, i^{\prime \prime}\right\}$, where $i^{\prime} \in W^{\prime}, i^{\prime \prime} \in W^{\prime \prime}$, with the $j$ th colour, $j=0,1, \ldots, k-1$, if $j$ appears at the $i^{\prime}$ th position in the expansion of $i^{\prime \prime}$ in the $k$-ary system. Note that for each pair of vertices $x^{\prime}, y^{\prime} \in W^{\prime}$ there exists $z^{\prime \prime} \in W^{\prime \prime}$ such that the edges $\left\{x^{\prime}, z^{\prime \prime}\right\}$ and $\left\{y^{\prime}, z^{\prime \prime}\right\}$ are coloured with different colours; it is enough to take $z^{\prime \prime}$ which has different digits at positions $x^{\prime}$ and $y^{\prime}$. Similarly, for every $x^{\prime \prime}, y^{\prime \prime} \in W^{\prime \prime}$ one can find a position $z^{\prime}$ at which the digits of the $k$-ary expansions of $x^{\prime \prime}$ and $y^{\prime \prime}$ differ; then the colours of the edges $\left\{x^{\prime \prime}, z^{\prime}\right\}$ and $\left\{y^{\prime \prime}, z^{\prime}\right\}$ must differ as well. Hence, every admissible colouring of the vertices of such a coloured graph must use different colours for different vertices, i.e. the $k$-chromatic number of the graph equals $n$.

The proof of the lower bound for $f_{k}(n)$ is slightly less immediate. Let $G$ be a graph with $n$ vertices and

$$
e(G) \leq n\left(\log _{k} n-4 \log _{k} \log _{k} n-5\right)
$$

edges, which are coloured with $k$ colours. We need to show that for each such colouring there exists an admissible colouring of the vertices of $G$ which uses only $n-1$ colours. 
Our argument will be based on the following observation (see Tuza [5]).

Claim. If the edges of the complete graph $K_{n}$ are covered by a family of $k$-partite graphs $G_{1}, \ldots, G_{m}$, of $r_{1}, \ldots, r_{m}$ vertices, respectively, then $\sum_{i} r_{i} \geq$ $n \log _{k} n$.

Let us partition the vertices of $G$ into two classes $W^{\prime}$ and $W^{\prime \prime}$, where $W^{\prime}$ consists of all vertices of $G$ of degree at least $\log _{k}^{2} n$. Then

$$
\left|W^{\prime}\right| \leq \frac{2 e(G)}{\log _{k}^{2} n}<\frac{2 n}{\log _{k} n},
$$

and so $\left|W^{\prime \prime}\right| \geq n\left(1-2 / \log _{k} n\right)$. Now, for every vertex $w^{\prime} \in W^{\prime}$ we define a complete $k$-partite graph $G_{w^{\prime}}$, choosing as the $i$ th set of the $k$-partition of $G_{w^{\prime}}$ the set of all vertices of $W^{\prime \prime}$ which are connected to $w^{\prime}$ by edges of the $i$ th colour. Thus, the total number of vertices of $G_{w^{\prime}}$ is the same as the number of neighbours of $w^{\prime}$ in $W^{\prime \prime}$. Furthermore, let $H^{\prime \prime}$ be the graph with vertex set $W^{\prime \prime}$, in which two vertices are adjacent if they lie within distance two in the subgraph $G\left[W^{\prime \prime}\right]$ induced by $W^{\prime \prime}$ in $G$. Note that the maximum degree $\Delta\left(H^{\prime \prime}\right)$ of $H^{\prime \prime}$ is bounded from above by $\Delta^{2}\left(G\left[W^{\prime \prime}\right]\right) \leq \log _{k}^{4} n$, and thus $H^{\prime \prime}$ can be decomposed into

$$
m^{\prime \prime} \leq\left\lceil\log _{k}\left(\log _{k}^{4} n+1\right)\right\rceil \leq 4 \log _{k} \log _{k} n+1
$$

$k$-partite subgraphs $H_{1}, \ldots, H_{m^{\prime \prime}}$. Observe also that the sum of vertices of all graphs $\left\{G_{w^{\prime}}\right\}_{w^{\prime} \in W^{\prime}}$ and $\left\{H_{i}\right\}_{i=1}^{m^{\prime \prime}}$ can be bounded from above by

$$
\begin{aligned}
\sum_{w^{\prime} \in W^{\prime}}\left|G_{w^{\prime}}\right| & +\sum_{i=1}^{m^{\prime \prime}}\left|H_{i}\right| \\
& \leq e(G)+\left|W^{\prime \prime}\right|\left(4 \log _{k} \log _{k} n+1\right) \\
& \leq\left|W^{\prime \prime}\right|\left(\frac{\log _{k} n-4 \log _{k} \log _{k} n-5}{1-2 / \log _{k} n}+4 \log _{k} \log _{k} n+1\right) \\
& <\left|W^{\prime \prime}\right|\left(\log _{k} n-1\right)<\left|W^{\prime \prime}\right| \log _{k}\left|W^{\prime \prime}\right| .
\end{aligned}
$$

Hence, the Claim implies that there exists a pair of vertices $\left\{x^{\prime \prime}, y^{\prime \prime}\right\}$ which appears as an edge in none of the graphs $\left\{G_{w^{\prime}}\right\}_{w^{\prime} \in W^{\prime}}$ and $\left\{H_{i}\right\}_{i=1}^{m^{\prime \prime}}$, i.e. vertices $x^{\prime \prime}$ and $y^{\prime \prime}$ are not adjacent, they have no common neighbours in $W^{\prime \prime}$, and for each of their common neighbour $z^{\prime} \in W^{\prime}$ both edges $\left\{x^{\prime \prime}, z^{\prime}\right\}$ and $\left\{y^{\prime \prime}, z^{\prime}\right\}$ are coloured with the same colour. Thus, the colouring in which $x^{\prime \prime}$ and $y^{\prime \prime}$ are 
coloured with the same colour, while all other vertices of $G$ receive different colours, is admissible. Consequently, $\chi_{k}(G) \leq n-1$ and the assertion follows.

Now let us return to Erdös's original question. Recall (see [2-4]) that the oriented chromatic number of a graph $G$ is defined as the smallest number $\ell$, such that for every orientation $\vec{G}$ of $G$ there exists a tournament $\vec{T}(\vec{G})$ on $\ell$ vertices such that $\vec{G}$ can be homomorphically embedded into $\vec{T}(\vec{G})$. Let $g(n)$ be the smallest number for which there exists a graph $G$ with $n$ vertices, $g(n)$ edges, and the oriented chromatic number $n$. It is not hard to see that $G$ has the above property if and only if in some orientation $\vec{G}$ of $G$ each pair of non-adjacent vertices of $G$ is connected by a directed path of length two. Hence, after a quick look at the proof of Theorem 1 for the case $k=2$, one can easily modify it and arrive at the following answer to Erdös's problem.

Theorem 2. If $n$ is large enough then

$$
n\left(\log _{2} n-4 \log _{2} \log _{2} n-5\right) \leq g(n) \leq\left\lceil\log _{2} n\right\rceil\left(n-\left\lceil\log _{2} n\right\rceil\right) .
$$

We conclude with a few words on the expected behaviour of $f_{k}(n)$ and $g(n)$. It is tempting to conjecture that the upper bounds given in Theorems 1 and 2 are close to the truth and $f_{k}(n)=n \log _{k} n+O(n)$ and $g(n)=n \log _{2} n+O(n)$, where the term $O(n)$ depends on the arithmetic properties of $n$. On the other hand, we should mention that the elementary bipartite construction we used to get the upper bound for $f_{k}(n)$ is far from being best possible. For instance, one can modify it slightly by deleting one vertex from the smaller set of the bipartition at the same time adding a perfect matching to the larger of the sets; the graph obtained in such a way has $k$-chromatic number $n-1$ and, typically, less than $\left\lceil\log _{k}(n-1)\right\rceil\left(n-1-\left\lceil\log _{k}(n-1)\right\rceil\right.$ edges. Furthermore, if we have a family of graphs $G_{1}, G_{2}, \ldots, G_{r}$, such that $G_{i}$ has $n_{i}$ vertices and the $k$-chromatic number $n_{i}$, then the graph $1+\sum_{i=1}^{r} G_{i}$ obtained by taking disjoint copies of $G_{1}, G_{2}, \ldots, G_{r}$ and adding to it one more vertex of degree $\sum_{i=1}^{r} n_{i}$ has the largest possible $k$-chromatic number as well. Hence, one can take small "extremal" graphs and build out of them graphs with a large $k$ chromatic number, which also improve the upper bound for $f_{k}(n)$ given in Theorem 1. The same observation applies for the oriented chromatic number; the Reader can easily provide examples of sparse small extremal graphs 
for this problem with $n$ vertices and less than $\left\lceil\log _{2} n\right\rceil\left(n-\left\lceil\log _{2} n\right\rceil\right)$ edges, and describe a recursive construction of obtaining such an extremal graph out of two smaller ones. However, to find an exact guess for the structure of the extremal graphs does not seem to be easy for us.

\section{References}

[1] N. Alon and T. H. Marshall, Homomorphisms of edge-coloured graphs and Coxeter groups, manuscript.

[2] A. V. Kostochka, E. Sopena and X. Zhu, Acyclic and oriented chromatic numbers of graphs, J. Graph Theory 24 (1997) 331-340.

[3] A. Raspaud and E. Sopena, Good and semi-strong colorings of oriented planar graphs, Information Processing Letters 51 (1994) 171-174.

[4] E. Sopena, The chromatic number of oriented graphs, J. Graph Theory 25 (1997) 191-205.

[5] Zs. Tuza, Intersection properties and extremal problems for set systems. In: "Irregularities of partitions" (G. Halász, V. T. Sós, Eds.), SpringerVerlag, 1989, pp.141-151. 